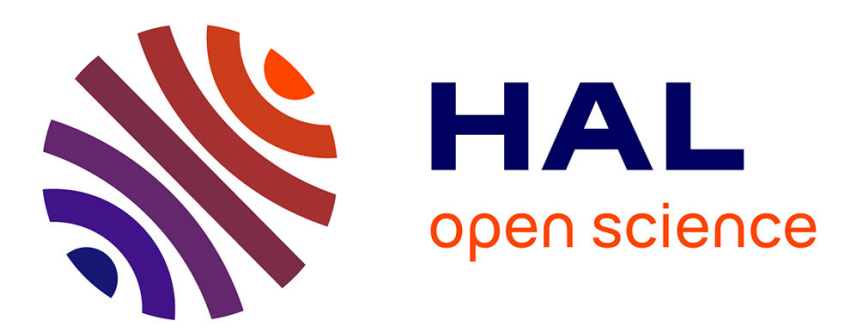

\title{
A Player Model for Adaptive Gamification in Learning Environments
}

Baptiste Monterrat, Michel Desmarais, Elise Lavoué, Sébastien George

\section{To cite this version:}

Baptiste Monterrat, Michel Desmarais, Elise Lavoué, Sébastien George. A Player Model for Adaptive Gamification in Learning Environments. AIED 2015, Jun 2015, Madrid, Spain. pp. 297-306. hal01165497

\section{HAL Id: hal-01165497 \\ https://hal.science/hal-01165497}

Submitted on 19 Jun 2015

HAL is a multi-disciplinary open access archive for the deposit and dissemination of scientific research documents, whether they are published or not. The documents may come from teaching and research institutions in France or abroad, or from public or private research centers.
L'archive ouverte pluridisciplinaire HAL, est destinée au dépôt et à la diffusion de documents scientifiques de niveau recherche, publiés ou non, émanant des établissements d'enseignement et de recherche français ou étrangers, des laboratoires publics ou privés. 


\title{
PREPRINT VERSION
}

\section{A Player Model for Adaptive Gamification in Learning Environments}

\author{
Baptiste Monterrat ${ }^{1,2,3}$, Michel Desmarais $^{4}$, Élise Lavoué ${ }^{5}$, Sébastien George ${ }^{6}$ \\ ${ }^{1}$ Woonoz compagny, 69009 Lyon, France \\ ${ }^{2}$ Université de Lyon, CNRS \\ ${ }^{3}$ INSA-Lyon, LIRIS, UMR 5205, F-69621, France \\ baptiste.monterrataliris.cnrs.fr \\ ${ }^{4}$ Polytechnique Montréal, Canada \\ michel. desmarais@polymtl.ca \\ ${ }^{5}$ Magellan, IAE Lyon, Université Jean Moulin Lyon 3, France \\ elise. lavoueduniv-lyon3. fr \\ ${ }^{6}$ LUNAM Université, Université du Maine, EA 4023, LIUM, 72085 Le Mans, France \\ sebastien.george@univ-lemans.fr
}

\begin{abstract}
Many learning environments are swiftly abandoned by the learners, even if they are effective. Gamification is as a recent game-based learning approach that can enhance the learners' motivation. However, individual expectations and preferences towards game-like features may be very different from one person to another. This paper presents a model to adapt gamification features according to a player profile of the learners. Two version of this model are evaluated within a gamified online learning environment. The first version comes from experts' judgment, and the second one is induced from empirical data. Our experiments confirm that the first version can be efficient to predict the player's preferences among the gamification features.
\end{abstract}

Keywords: Gamification, Adaptation, Web-based learning, Motivation, Player Model.

\section{Introduction}

Research in the field of game-based learning aims at making the learning activities more fun and more engaging for the users by proposing two main approaches: learning games and gamification. Learning games, often named "serious games", refer to the use of games for learning purposes [1]; gamification relies instead on game design elements embedded in a learning environment to foster student motivation [2]. Turning the learning environment into a serious game requires a complete redesign, which could be very expensive and time consuming. In this paper, we focus on gamification in order to integrate gaming features in already existing learning environments.

More specifically, we address the problem of motivation in a web-based learning environment within which the learner is guided by the system. In this type of environment, the absence of a human tutor often causes a lack of learner's interest.

Our approach relies on gamification where we integrate game mechanics to existing learning environments, such as scoreboards, rewards, and other fun features. This 


\section{PREPRINT VERSTON}

approach is generally easier to realize than to turn the whole learning experience into a game.

The word "gamification" has been introduced in early 2010. This approach is used in various fields such as marketing, health, and crowdsourcing. In this work we are interested in the use of gamification in education [3]. Games have always been used for learning, but the arrival of the gamification perspective brought major changes in the way we study games for learning. Some even consider gamification as a new educational theory [4], alongside of behaviorism, cognitivism, constructivism and connectivism.

Gamification has been proven to be effective in numerous situations [5], but little is known about adaptivity for gamification. Most gamified systems integrate the game elements under a "one size fits all" approach, without means to adapt the learning process or the learning environment. whereas it has long been recognised than individualised learning is much more efficient than classroom learning [6], we believe the same principles are at stake in gamification: one means of gamifying does not fit to all users.

The research question studied in this paper is the following: "How to adapt the game elements of a learning environment to learners according to their player types?" A review of gamification and player models for adaptation is presented is section 2 . The model we propose for adaptive gamification is presented in section 3. Section 4 presents two experimental evaluations of various versions of the model. One model is based on human expertise, and the others rely on empirical data. Section 5 concludes on the validity of the experts based model.

\section{Gamification and Player Profiles}

In this section we first review common gamification elements, and then review various player profiles by which adaptation can be derived. Finally we study adaptation techniques and models that can help to relate the gamification elements with the player profiles factors.

\subsection{Gamification Elements}

Game elements are at the core of gamification and they can be categorized from the more abstract to the more concrete ones. Deterding et al. [2] propose five levels of abstraction. The game design methods come first, e.g. playtesting. Secondly come the game models like engagement loops and feedback loops. At the third level are the game design principles and heuristics, including setting clear goals and a variety of game styles. One of the most popular principle relies on the balance between challenge and player skills in order to reach the state of flow [7]. At the fourth level are the game mechanics such as the use of time constraints, points systems, and limited resources. Finally, those game mechanics are reified by interface elements. Among the most common game elements are the following: 


\section{PREPRINT VERSION}

- Points counter

- Badges (trophies symbolizing a task accomplishment)

- Leaderboards

Those elements rely on quantifying the user's activity in order to reward it. In this paper we place adaptation on the game mechanics level. We will refer to "game features" as a game mechanic emerging from one or more user interface elements. As an example, a game feature can be a points counter associated with some badges to reward a given score.

\subsection{Player Typologies}

Players generally have favorite types of games, and they feel engaged with some game mechanics but not all of them. Bartle's classification in four player types [8] is well known (killer, achiever, explorer, socializer), but it does not reflect diversity of player types that has been highlighted in several works more recently. For example, Yee [9] identifies three main motivational components (achievement, social interaction and immersion). A review of player types studies by Ferro et al. [10] distinguishes five player types (dominant, objectivist, humanist, inquisitive and creative). As they focus on personalized gamification (rather than games), they relate player types directly to game elements and mechanics. One of the most recent contributions in this area is the BrainHex gamer typology [11]. This classification includes seven player types based on insights from neurological findings: seeker, survivor, daredevil, mastermind, conqueror, socializer and achiever. Contrary to previous typologies, the BrainHex one is not related to a specific game genre like MMORPG. In addition, it is backed by an online survey that was taken by more than 60,000 players. For these reasons, we selected this classification as a player model for an adaptive gamification.

\subsection{Adaptation of Games and Gamification}

Adaptation in games. A review of sixteen game adaptation techniques designed between 2002 and 2009 [12] focused on both entertainment games and serious games. A large majority of these techniques adjusts the difficulty level of the activity under various forms: they adapt the opponent's behavior, the game speed, the scenario or the feedback, mostly with the intent to prevent the game from being too difficult or too easy. The rest of the works studied in this review propose a generic adaptation of game parameters values according to player satisfaction, or adapt the learning part of the games rather than the gaming part.

Only one study addresses the difference in the game mechanics by proposing a scenario adapted to the personality of the players: the work of Natkin et al. [13]. The personality types are based on the Five-Factor Model (ibid.), and are used to select diverse quests for the players, like defeating other players or solving puzzles. Their system allows the use of various game dynamics for various players. We aim at applying a similar approach to existing learning environments by using game features instead of quests. 


\section{PREPRINT VERSION}

Models for gamification. The adaptation of gamification is still in its infancy, but some models identify the concepts we can rely on. In order to adapt game features to players, we need to establish their links with the a player typology. Robinson and Bellotti [14] present one of the first taxonomies of gamification elements. They are classified in 6 categories, such as social elements and status information. Sailer [15] proposes a list of game elements and describes their links to various motivational concepts. These two contributions help understanding how the game features can influence player motivation, but do not show how to relate them to player profiles.

Zichermann and Cunningham [16] propose to use the MDA model (Mechanics, Dynamics, Aesthetics) developed by Hunicke et al. [17]. In this framework, mechanics refer to game elements in the user interface. Dynamics are at a higher level of abstraction, they refer to the interactions between the mechanics and the player. Finally, aesthetics describe the emotional response of the player to the experienced dynamics. This framework can help to relate game features to dynamics, and then to link the dynamics to the players who are receptive to those emotions. However, introducing more levels of abstraction between the game features and the player may multiply the risk of mistakes.

In this paper we propose a method to relate directly the game features to player types, without considering intermediate concepts.

\section{$3 \quad$ Player Model for Adaptive Gamification}

Our approach to the adaptive interface of the learning environment relies on two separate engines developed independently, one for the didactic content adaptation and the other for the gamification adaptation [18]. The didactic adaptation takes into account the learner's knowledge state, whereas the gamification adaptation takes into account a gamer profile based on the BrainHex classification. The didactic content engine chooses the items to present to the learner, whereas the gamification engine adapts the interface game features displayed on the interface. In this paper, we only focus on the gamification engine. The game features are implemented in such a way as to be toggled on or off independently of the didactic content engine and the general workflow.

\subsection{Player Adaptation Model}

In order to adapt the game features to the learner profile, we need a model to estimate the quality of fit of the game features to a player/student profile. We developed a model that estimates the preference for a feature by a weighted sum of personality traits, which bears similarities to existing learner models that predict student success based on a linear or boolean combination of skills [19].

Assume a matrix $\mathbf{B}$ of size $m \times k$ that represents the $k$ traits of $\mathrm{m}$ users, and a matrix A of size $k \times n$ that represents the weights of the $k$ for each of the $n$ game features. Then, the product of these two matrices represents the expected preferences of the $m$ users for the $n$ game features: $\mathbf{R}=\mathbf{B} \mathbf{A}$. 


\section{PREPRINT VERSION}

\begin{tabular}{|c|c|c|c|c|c|c|c|}
\hline & $\mathrm{f} 1$ & $\mathrm{f} 2$ & f3 & & \multicolumn{3}{|c|}{ C } \\
\hline $\mathrm{u} 1$ & 10 & 00 & 05 & & $\mathrm{u} 1$ & 10 & 00 \\
\hline u2 & 00 & 06 & 12 & $=$ & $\mathrm{u} 2$ & 00 & \\
\hline u3 & 06 & 03 & 09 & & u3 & 06 & \\
\hline $\mathrm{u} 4$ & -08 & 03 & 02 & & $\mathrm{u} 4$ & -08 & \\
\hline
\end{tabular}

Fig. 1. An example of linear model $\mathbf{R}=\mathbf{B}$ A. This example comprises 4 users (u1-u4), 3 game features (f1-f3) and a 2-factors player model: competition (C) and social (S).

An example of this simple linear model is given in Figure 1. The preferences of users $u l$ to $u 4$ for features $f 1$ to $f 3$ are the product of the users' score over two traits, competitor $(C)$ and socializer $(S)$, and the weights of each trait for the three different features. For example, for $f 3$, the $C$ trait has a 0.5 weight and the $S$ trait a weight of 1 . Taking the scores of $\mathrm{u} 4$ on these respective traits yields the following result for $\mathrm{f} 3$ : $0.5 \times-8+1 \times 6=2$. Taking the vector of scores for $\mathrm{u} 4$ indicates that feature $\mathrm{f} 1$ is proscribed with a value of -8 , whereas features $\mathrm{f} 2$ and $\mathrm{f} 3$ have a relatively close score of 2 and 3 respectively.

Figure 1 example contains only two traits. In this work, we use the BrainHex gamer profile, which contains 7 traits as explained in section 2.2. This model allows a player to be represented on this vector of traits with values in the range $[-10,20]$. Therefore, the $\mathbf{B}$ matrix for our study has 7 columns and the negative values are values over the traits can result in negative values in matrix $\mathbf{R}$, which are indicative of a feature misfit which can result in a negative motivational impact.

\subsection{Estimating Matrix A}

Whereas matrix $\mathbf{B}$ can be directly obtained from the answers to the BrainHex questionnaire over the 7 traits of the gamer profile, matrix $\mathbf{A}$ must be derived by some other means. Two such means are considered in this study:

Human expert derived (1). The BrainHex traits were designed within the space of gaming motivational factors. Therefore, it becomes relatively straightforward for an expert to intuitively estimate what motivational factors can be involved in some of the features, such as the socializer type who will strive for features that allows interaction with other users, or the conqueror who will prefer features that can provide a measure of progress towards some end.

Empirically derived from observed data (2). If a matrix $\mathbf{R}$ can be obtained either by some means to measure the preference of students for the features, or through observation or through direct questioning, and that the $\mathbf{B}$ matrix is provided by the BrainHex questionnaire, then the matrix $\mathbf{A}$ can be estimated by the least squares method through the following equation:

$$
A=\left(B B^{T}\right)^{-1} B^{T} R
$$




\section{PREPRINT VERSION}

In our study, we asked the students to rate each feature. These ratings are an estimate for matrix $\mathbf{R}$, with which the above equation is used to estimate matrix $\mathbf{A}$ in return.

Both approaches have their inherent advantages and caveats. The expert derived matrix is prone to subjective biases, but it is straightforward for experts to estimate it. The empirically derived matrix is more objective and potentially more accurate, but only under the condition that sufficient data is gathered. We conducted experiments to investigate these questions.

\section{$4 \quad$ Implementation and Evaluation}

We first conducted an experiment to investigate the effectiveness of the expert derived vs. the empirically derived A-matrices. In a later experiment, we chose the expert derived A-matrix to adapt game features and assess the quality of the adaptation it provides to the learners.

\subsection{Learning Environnement and Games Features}

Let us first describe in more details the learning environment within which the gamification adaptation takes place, named Projet Voltaire ${ }^{1}$ and developed by Woonoz Inc. The environment is designed to learn and memorize the French grammar rules. As mentioned earlier, a content engine adapts the difficulty of the learning material by taking into account the history of success outcome of the learner. Combined to this content adaptation, five game features are also available.

All the game features implement different game mechanics. The first feature is a set of stars lighted by the players when a grammar rule is learnt. The second feature is a leader board that reports the number of consecutive correct answers and the first name of the students who are close to this score. The user can appreciate through this feature whether s/he is getting ahead or behind the neighbouring student on this board. The third feature provides users with a way to give tips to each other, in order to better understand the grammar rules. It aims at enhancing social interactions. The fourth feature represents a walker climbing a mountain. Some flags are placed on the way, they give the user an access to anecdotes about French spelling. The last feature is a timer that encourages the user to move quickly.

\subsection{Experiment 1: Estimating Matrix A}

Experiment Settings. 140 users participated to the first experiment. They had to answer a survey before using Projet Voltaire for three weeks. After this period, they had to answer a second survey. The first survey contained the BrainHex test ${ }^{2}$, which provided us with the full BrainHex profile of the users: seven values in $[-10,20]$. This

1 The Projet Voltaire, developed by Woonoz, is available on projet-voltaire.fr.

2 The BrainHex test, developed by International Hobo, is available on brainhex.com. 


\section{PREPRINT VERSION}

data provided the B matrix. During the three weeks of use of Projet Voltaire, only two of the five game features were activated avoid overloading the interface. The two game features were randomly selected among the five available features. In the second survey we asked the users if they felt the game features were a motivating factor. The answers were used to build the $\mathbf{R}$ matrix.

As each user only had two game features, their answers are not sufficient to complete the $\mathbf{R}$ matrix: there are three missing values on each line. In order to fill the blanks, we follow the recommendation of Ayers at al. [20] to obtain values with minimal information: the value between the mean of the column and the mean of the row. In the $\mathbf{R}$ matrix, the mean of the column indicates the global motivational effect of the game feature on all users. The mean of the row indicates whether the user tends to give high or low values. The ratings of the $\mathrm{R}$ matrix was thereafter used to calculate the empirical A-matrix.

Human Expert A-Matrix (1). Six experts ${ }^{3}$ were asked to fill an A-matrix of weights for mapping BrainHex categories to game features. They first learnt the BrainHex classification. They then used Projet Voltaire for about one hour, interacting with all the five game features. Finally they had to provide a value to link each feature (5) to each player type (7), and thus build a 7 x 5 A-matrix. The values could be chosen among the following:

1. Very strong match: 1

2. Strong match: 0.75

3. Medium match: 0.5

4. Weak match: 0.25

5. No match: 0

To get a single A-matrix from the six experts, we took the median of the six judgements for each of the 35 values (see Table 1). The median is a way to get the value most experts will agree with, and it prevents the result from being influenced by an extreme value. The resulting A-matrix was used in the formula $\mathrm{R}=\mathrm{B}$ A to obtain the R-expert predictions.

Table 1. Experts A-matrix. Columns: game features. Rows: BrainHex player types.

\begin{tabular}{rccccc}
\hline & Stars & Leader board & Tips & Walker & Timer \\
\hline Seeker & 0.5 & 0 & 0.75 & 0.88 & 0 \\
Survivor & 0.13 & 0.5 & 0 & 0 & 0.38 \\
Daredevil & 0.63 & 0.63 & 0 & 0.13 & 0.88 \\
Mastermind & 0.63 & 0.63 & 0.38 & 0.25 & 0.25 \\
Conqueror & 0.75 & 1 & 0.13 & 0.38 & 0.75 \\
Socializer & 0.13 & 0.13 & 1 & 0.25 & 0 \\
Achiever & 1 & 0.75 & 0.13 & 0.88 & 1
\end{tabular}

3 The experts are academics specialised in serious games and gamification. 


\section{PREPRINT VERSION}

In order to confirm the validity of the R-experts matrix, we measured the experts' agreement with IntraClass Correlation (ICC) [21] and obtained a value of 0.43 . This is considered as a moderate value but high enough to confirm the agreement between experts.

Users' Data A-matrix (2). To estimate the A matrix, a least square method of equation (1) can be applied given $\mathbf{R}$ and $\mathbf{B}$. Estimation of matrix $\mathbf{A}$ is done through an eight-fold cross validation. The training set is used on the $7 / 8$ of the data to estimate A. The missing values in $\mathbf{R}$ have been replaced by the expected values (column-row average). The remaining $1 / 8$ of the data is used for the testing phase where we use the estimated A matrix to predict learner preferences. The training set rotated seven times to cover all the data. We refer to the obtained prediction as R-data.

Results and Discussion. The predictions R-experts and R-data have been compared to the real values provided by the users ( $2 / 5$ values of $\mathbf{R})$ to be evaluated. If a prediction is correct, the high values of the predicted $\mathrm{R}$ will match to the high values of the real $\mathrm{R}$, and the same goes for the low values. Accordingly we evaluated the predictions with a linear correlation between the predicted and real values. The correlation coefficient between the values of $\mathbf{R}$ and $\mathbf{R}$-experts is $r=0.2207$. The correlation coefficient between the values of $\mathbf{R}$ and $\mathbf{R}$-data is $r=0.1822$.

Two factors led us to choose the experts derived A-matrix over the empirical data derived A-matrix. Firstly, the agreement among the six experts (ICC) provides some validity to the experts matrix. Secondly, the prediction of the experts has a better correlation with the answers provided by the users. Accordingly the experts A-matrix was selected for the second experiment.

\subsection{Experiment 2 : Adaptation of Game Features}

The second experiment consisted in using matrix $\mathbf{A}$ from the experts to adapt the game features based on the learners' BrainHex profile obtained from the questionnaire.

Experiment Settings. 280 users participated to this second experiment, after being divided into two groups. As in the experiment 1 they filled in the BrainHex survey, then got access to Projet Voltaire for three weeks, and answered a game feature assessment questionnaire. Before they started using Projet Voltaire, we used the BrainHex results (B) and the experts matrix (A) to predict the motivational impact of the five game features on each user $(\mathbf{R})$. The members of Group 1 were provided with the two game features that best matched their profile, and the members of Group 2 with the two features that worse matched their profile. 


\section{PREPRINT VERSION}

Results and Discussion. The second experiment revealed two interesting results regarding the choice of the A-matrix.

Firstly, the members of Group 1 with adapted features spent on average 2 hours and 38 minutes on Projet Voltaire, whereas the members of Group 2 with counteradapted features spent on average 1 hour and 54 minutes on the environment. Thus members of Group 1 spent $39 \%$ more time on the learning environment. A Student- $t$ test over this difference reveals a p-value of 0.0426 , and confirms that the adaptation model relying on the experts matrix has indeed a positive impact on the learners engagement at $\mathrm{p}<0.05$.

Secondly, the members of Group 1 and 2 gave similar values for the evaluation of their game features. To the item "This feature is motivating for me" (on a scale from 1 to 7), the mean value given by Group 1 is 4.58 , whereas it is 4.55 for Group 2. This result shows that the users seem not aware of the impact of the adapted features on their engagement.

\section{$5 \quad$ Conclusion and Perspectives}

In this paper we presented an adaptation model to provide learners with game features that match to their player profile. It is based on a linear relation between game features and player types. The model is generic, but relies on an association matrix that is specific to the game features implemented in the learning environment. This matrix can rely on experts' judgment or empirical data. An experiment has proven the effectiveness of the experts-based matrix, as the members of the group with adapted features spent 39\% more time on the learning environment than the members of the group with counter-adapted features. The model based on empirical data could not be validated in this experiment for two reasons. First this type of model requires a large amount of users, which was not sufficient here. Then the model has to rely on a variable that is significantly impacted by the level of matching between the game features and the user, which seems not to be the case with the values provided consciously by the users.

At present, the adaptation is made possible thanks to the BrainHex test answered by the learners before using the learning environment. The next step of our work will be to update the model in real time according to the users' interactions. For example, if a user disables a game feature, then s/he's probably not responsive to its game mechanics.

Acknowledgements. We thank the ANRT and Woonoz for funding this research work. We also would like to thank Woonoz for the development of the experimental version of Projet Voltaire and for linking us to their users who accepted to participate in our experiments. We also thank the experts for their work on the A-matrix. Finally we thank International Hobo for providing us with the BrainHex test. 


\section{PREPRINT VERSION}

\section{References}

1. Prensky, M.: Digital game-based learning. New York: McGraw-Hill (2001)

2. Deterding, S., Dixon, D., Khaled, R., \& Nacke, L.: From game design elements to gamefulness: defining gamification. In Proceedings of the 15th International Academic MindTrek Conference: Envisioning Future Media Environments, p. 9-15 (2011)

3. Kapp, K. M.: The Gamification of Learning and Instruction: Game-based Methods and Strategies for Training and Education. John Wiley \& Sons (2012)

4. Bíró, G. I.. Didactics 2.0: A Pedagogical Analysis of Gamification Theory from a Comparative Perspective with a Special View to the Components of Learning (2014).

5. Hamari, J., Koivisto, J., \& Sarsa, H.: Does Gamification Work?-A Literature Review of Empirical Studies on Gamification. In Proceedings of the 47th Hawaii International Conference on System Sciences (2014)

6. Bloom, B. S.: The 2 sigma problem: The search for methods of group instruction as effective as one-to-one tutoring. Educational researcher, 4-16 (1984)

7. Csikszentmihalyi, M.: Finding flow: The psychology of engagement with everyday life. Basic Books (1998)

8. Bartle, R.: Richard A. Bartle: Players Who Suit MUDs (1996)

9. Yee, N.: Motivations for play in online games. CyberPsychology \& Behavior, 9(6), 772-775 (2006)

10. Ferro, L. S., Walz, S. P., \& Greuter, S.: Towards personalised, gamified systems: an investigation into game design, personality and player typologies. In Proceedings of The 9th Australasian Conference on Interactive Entertainment: Matters of Life and Death p. 7 (2013)

11. Nacke, L. E., Bateman, C., \& Mandryk, R. L.: BrainHex: A neurobiological gamer typology survey. Entertainment Computing, 5(1), 55-62 (2014)

12. Hocine, N., Gouaïche, A., Di Loreto, I., \& Abrouk, L.: Techniques d'adaptation dans les jeux ludiques et sérieux. Revue d'intelligence artificielle, 25(2), 253-280 (2011)

13. Natkin, S., Yan, C., Jumpertz, S., \& Market, B. Creating Multiplayer Ubiquitous Fames Using an Adaptive Narration Model Based on a User's Model. In Digital Games Research Association International Conference (2007).

14. Robinson, D., \& Bellotti, V.: A preliminary taxonomy of gamification elements for varying anticipated commitment. In Proc. ACM CHI 2013 Workshop on Designing Gamification: Creating Gameful and Playful Experiences (2013)

15. Sailer, M.: Psychological Perspectives on Motivation Through Gamification. Interaction Design and Architecture(s) Journal - IxD\&A, p. 28-37 (2013)

16. Zichermann, G., \& Cunningham, C.: Gamification by Design: Implementing game mechanics in web and mobile apps. O'Reilly Media, Inc (2011)

17. Hunicke, R., LeBlanc, M., \& Zubek, R.: MDA: A formal approach to game design and game research. Proceedings of the AAAI Workshop on Challenges in Game AI 04-04 (2004)

18. Monterrat, B., Lavoué, É., \& George, S.: Motivation for Learning - Adaptive Gamification for Web-based Learning Environments. In 6th International Conference on Computer Supported Education (2014) p. 117-125

19. Desmarais, M. C., \& Naceur, R.: A Matrix Factorization Method for Mapping Items to Skills and for Enhancing Expert-Based Q-Matrices. In Artificial Intelligence in Education (2013) p. 441-450

20. Ayers, E., Nugent, R., \& Dean, N.: A Comparison of Student Skill Knowledge Estimates. International Working Group on Educational Data Mining (2009)

21. Shrout, P. E., \& Fleiss, J. L.: Intraclass correlations: uses in assessing rater reliability. Psychological bulletin, (1979) 86(2), 420 